\title{
Nonexistence of solutions for quasilinear hyperbolic inequalities
}

\author{
Suping Xiao ${ }^{1}$ and Zhong Bo Fang ${ }^{2^{*}}$ (D)
}

\author{
${ }^{*}$ Correspondence: \\ fangzb7777@hotmail.com \\ ${ }^{2}$ School of Mathematical Sciences, \\ Ocean University of China, Qingdao \\ 266100, P.R. China \\ Full list of author information is \\ available at the end of the article
}

\begin{abstract}
In this paper, we study the Cauchy problems for quasilinear hyperbolic inequalities with nonlocal singular source term and prove the nonexistence of global weak solutions in the homogeneous and nonhomogeneous cases by the test function method
\end{abstract}

MSC: 35L72; 35L15; 35B53

Keywords: Quasilinear hyperbolic inequality; Nonlocal singular source; Test function; Nonexistence

\section{Introduction}

In this paper, we consider the Cauchy problems for quasilinear hyperbolic homogeneous and nonhomogeneous inequalities with nonlocal singular source term of the forms, respectively,

$$
\begin{aligned}
& u_{t t} \geq \Delta u^{m}+|x|^{\alpha}|u|^{q}\left\|\beta^{\frac{1}{r}}(x)|u|\right\|_{r}^{s}, \quad(x, t) \in S_{\infty}, \\
& u_{t t} \geq \Delta u^{m}+|x|^{\alpha}|u|^{q}\left\|\beta^{\frac{1}{r}}(x) \mid u\right\|_{r}^{s}+\omega(x), \quad(x, t) \in S_{\infty},
\end{aligned}
$$

subject to the initial condition

$$
\left(u(x, 0), u_{t}(x, 0)\right)=\left(u_{0}(x), u_{1}(x)\right), \quad x \in R^{N},
$$

where

$$
\begin{aligned}
& S_{\infty}=R^{N} \times(0, \infty), \quad m, q, r, s>0, \quad \alpha>\frac{s \sigma}{r}, \\
& r(q+s)>\max \{1, m\}(r+s),
\end{aligned}
$$

the initial functions $u_{0}, u_{1} \in L_{\text {loc }}^{1}\left(R^{N}\right)$ are nonnegative, the weight function $\beta$ is positive and singular at the origin, that is, there exist constants $c>0$ and $\sigma \in R_{+}$such that

$$
\beta(x) \geq c|x|^{-\sigma}>0, \quad x \in R^{N} \backslash\{0\},
$$

and the nonhomogeneous term $w \in L_{\text {loc }}^{1}\left(R^{N}\right)$ is nonnegative.

(c) The Author(s) 2021. This article is licensed under a Creative Commons Attribution 4.0 International License, which permits use, sharing, adaptation, distribution and reproduction in any medium or format, as long as you give appropriate credit to the original author(s) and the source, provide a link to the Creative Commons licence, and indicate if changes were made. The images or other third party material in this article are included in the article's Creative Commons licence, unless indicated otherwise in a credit line to the material. If material is not included in the article's Creative Commons licence and your intended use is not permitted by statutory regulation or exceeds the permitted use, you will need to obtain permission directly from the copyright holder. To view a copy of this licence, visit http://creativecommons.org/licenses/by/4.0/. 
The existence and nonexistence of global and nonglobal solutions for nonlinear evolution equations (or inequalities) have been assiduously investigated during the past decades. The nonexistence of global solutions is a nonlinear Liouville-type theorem, and thus we can prove some properties of solutions in a bounded domain, which is a kind of essential reflection of blowup or singularity theory as well; see [1]. In this paper, we would like to investigate the nonexistence of global solutions of the Cauchy problems for nonlocal hyperbolic differential inequalities.

For the model

$$
u_{t t}-\Delta u=|u|^{p}, \quad(x, t) \in S_{T},
$$

this problem was first studied by John [2]. The author proved that if $1<p<1+\sqrt{2}$ and $N=3$, then all local solutions to (1.5), (1.3) with $u_{0}, u_{1} \in C_{0}^{\infty}\left(R^{3}\right)$ blow up at a finite time. Later, Glassy [3] showed the nonexistence of global solution for the critical value $p=1+$ $\sqrt{2}$ under the additional assumption that the initial values $u_{0}$ and $u_{1}$ both have positive average. Presumably, then there exists a critical value of $p$, say $p_{0}(N)$, such that the existence of all small global solutions holds if $p>p_{0}(N)$, whereas the nonexistence of most global solutions holds if $1<p<p_{0}(N)$; the critical value $p_{0}(N)$ satisfies

$$
p_{0}(N)=\infty, \quad N=1 ; \quad p_{0}(N)=\frac{1}{2(N-1)}\left[N+1+\sqrt{N^{2}+10 N-7}\right], \quad N=2,3 .
$$

Recently, Strauss and Tsutaya [4] proved that in case $N=3, u_{0} \equiv 0$, and $u_{1}(x) \geq C(1+$ $\left.|x|^{2}\right)^{-\frac{1+k}{2}}$, the problem has no global solution for any $p>1$ and any $k<\frac{2}{p-1}$. The critical case $k=\frac{2}{p-1}$ was treated in [5], where the global existence is proved in three space dimensions for $p>1+\sqrt{2}$ and for small data $u_{0} \in C^{3}\left(R^{3}\right)$ and $u_{1} \in C^{2}\left(R^{3}\right)$ satisfying $D^{\alpha} u_{0}(x), D^{\beta} u_{1}(x)=$ $O\left(|x|^{-1-k}\right)$ as $|x| \rightarrow \infty,|\alpha| \leq 3,|\beta| \leq 2$. Pohozaev and Veron [6] considered the quasilinear differential inequalities

$$
u_{t t}-L_{m}(\varphi(u)) \geq h(x)|u|^{p}, \quad(x, t) \in S_{\infty}
$$

where $h(x) \equiv 1, \varphi$ is a locally bounded real-valued function that satisfies $|\varphi(r)| \leq C|r|^{q}$ $(\forall r \in R)$ for some $C>0$ and $q>0$, and $L_{m}(\zeta)=\sum_{|\alpha|=m} D^{\alpha}\left(a_{\alpha}(x, t) \zeta\right)$ is a homogeneous differential operator of order $m$ in which the $a_{\alpha}$ are merely bounded measurable functions. They proved that there is no weak global solution to (1.6) with $\int_{R^{N}} u_{1}(x) d x \geq 0$ if $p>$ $\max (1, q)$ and either $2 N-m \leq 0$, or $2 N-m>0$ and $N \frac{(p-q)}{p+1} \leq \frac{m}{2}$. No assumption on the sign of the average of $u_{0}$ (which may not be integrable) or on its support are required. Later, Guedda [7] obtained the nonexistence of local and global solutions by the test function method.

For the study of the problem with nonhomogeneous term, Laptev [8] considered the semilinear hyperbolic-type inequalities with nonhomogeneous term

$$
u_{t t}-\triangle u \geq|u|^{p}+\omega(x), \quad(x, t) \in K \times(0, \infty)
$$

where $K$ is a conic domain. They proved that there is no nontrivial global solution by the test function method. 
Motivated by the above works, we can find that the study on nonexistence of global solutions for the quasilinear hyperbolic type inequalities (1.1) and (1.2) with nonlocal singular source term has not been started yet. The purpose of this paper is finding the influence of nonlocal singular source term and nonhomogeneous term on the nonexistence of nontrivial global weak solution by the test function method developed in [8, 9]. This method has two merits. Firstly, it is simple and accurate. In fact, the nonexistence of global solution is transformed into algebraic inequality analysis. Secondly, the comparison results and energy functional are not required, so it can be widely applied to nonlinear differential inequalities. There is no regularity assumption about initial data, and the range of parameters is completely different in the homogeneous and nonhomogeneous cases in the paper.

The rest of our paper is organized as follows. In Sect. 2, we introduce some definitions and main results of solutions for the homogenous and nonhomogenous problems. The proofs of the main results are given in Sect. 3.

\section{Preliminaries and main results}

Due to the degeneracy or singularity of equation (1.1) as $m>0$, problems (1.1), (1.3) and (1.2), (1.3) have no classical solution in general. Thus we give some definitions of a weak solution.

Definition 1 A nonnegative function $u(x, t)$ is called a weak solution of homogeneous problem (1.1), (1.3) in $S_{\infty}$ if the following conditions hold:

(i) $u, u^{m},|x|^{\alpha}|u|^{q}\left\|\beta^{\frac{1}{r}}(x)|u|\right\|_{r}^{s} \in L_{\text {loc }}^{1}\left(S_{\infty}\right)$,

(ii) for any nonnegative function $\zeta \in C_{0}^{2,2}\left(S_{\infty}\right)$, we have

$$
\begin{aligned}
& \iint_{S_{\infty}}|x|^{\alpha}|u|^{q} \zeta\left\|\beta^{\frac{1}{r}}(x)|u|\right\|_{r}^{s} d x d t+\int_{R^{N}} u_{1}(x) \zeta(x, 0) d x \\
& \quad \leq-\iint_{S_{\infty}} u^{m} \Delta \zeta d x d t+\iint_{S_{\infty}} u \zeta_{t t} d x d t+\int_{R^{N}} u_{0}(x) \zeta_{t}(x, 0) d x .
\end{aligned}
$$

Similarly, a nonnegative function $u(x, t)$ is called a weak solution of nonhomogeneous problem (1.2), (1.3) in $S_{\infty}$ if the following inequality holds:

$$
\begin{gathered}
\iint_{S_{\infty}}|x|^{\alpha}|u|^{q} \zeta\left\|\beta^{\frac{1}{r}}(x)|u|\right\|_{r}^{s} d x d t+\int_{R^{N}} u_{1}(x) \zeta(x, 0) d x+\iint_{S_{\infty}} \omega(x) \zeta d x d t \\
\leq-\iint_{S_{\infty}} u^{m} \Delta \zeta d x d t+\iint_{S_{\infty}} u \zeta_{t t} d x d t+\int_{R^{N}} u_{0}(x) \zeta_{t}(x, 0) d x .
\end{gathered}
$$

Our main results are as follows.

\section{Theorem 1 If}

(i) either $N=1$, or

(ii) $N>1, N \leq \frac{2 r(q+s+1)+(1+m)(r \alpha-s \sigma)}{2 r(q+s-m)-(1+m) s}$, then any nonnegative weak solution to homogeneous problem (1.1), (1.3) has no nontrivial global solution. 
Remark 1 In fact, if we choose $m=1$ in Theorem 1, then we get that the homogeneous problem (1.1), (1.3) has no global solution if either $N=1$ or $1<N \leq 1+\frac{1}{q}$, which improves the results of Pohozaev and Veron [6].

Theorem 2 Let $u(x, t)$ be a solution of the homogeneous problem (1.1), (1.3). If the initial data $u_{1}$ are compactly supported, then

$$
\begin{aligned}
& \int_{0}^{2^{\frac{1}{k}} \rho^{\frac{2}{k}}} \int_{R^{N} \cap\left\{|x| \leq 2^{\frac{1}{\mu}} \rho^{\frac{2}{\mu}}\right\}}|x|^{\alpha}|u|^{q} \zeta\left\|\beta^{\frac{1}{r}}(x)|u|\right\|_{r}^{s} d x d t+\int_{R^{N} \cap\left\{|x| \leq 2^{\frac{1}{\mu}} \rho^{\frac{2}{\mu}}\right\}} u_{1} \zeta(x, 0) d x \\
& \quad \leq C \rho^{\frac{2}{k}+\left(-\frac{4}{\mu} \sigma_{4}+\frac{2}{\mu}\left(\frac{s \sigma \sigma_{4}}{r \sigma_{3}}-\frac{\alpha \sigma_{4}}{\sigma_{3}}\right)+\frac{2 N}{\mu}\right) \frac{\sigma_{3}^{\prime}}{\sigma_{4}}} .
\end{aligned}
$$

Indeed, retaining only the first summand on the left-hand side of the inequality in Theorem 2, we obtain that

$$
\int_{R^{N} \cap\left\{|x| \leq 2^{\frac{1}{\mu}} \rho^{\frac{2}{\mu}}\right\}} u_{1} \zeta(x, 0) d x \leq C \rho^{\frac{2}{k}+\left(-\frac{4}{\mu} \sigma_{4}+\frac{2}{\mu}\left(\frac{s \sigma \sigma_{4}}{r \sigma_{3}}-\frac{\alpha \sigma_{4}}{\sigma_{3}}\right)+\frac{2 N}{\mu} \frac{\sigma_{3}^{\prime}}{\sigma_{4}}\right.}
$$

If $\frac{2}{k}+\left(-\frac{4}{\mu} \sigma_{4}+\frac{2}{\mu}\left(\frac{s \sigma \sigma_{4}}{r \sigma_{3}}-\frac{\alpha \sigma_{4}}{\sigma_{3}}\right)+\frac{2 N}{\mu}\right) \frac{\sigma_{3}^{\prime}}{\sigma_{4}}<0$, then the right-hand side obviously tends to zero as $\rho \rightarrow \infty$, whereas the left-hand side is increasing (more precisely, it is nondecreasing if the initial data $u_{1}$ are compactly supported). Therefore, for any initial data, there is $\rho_{0}$ such that this inequality does not hold for $\rho>2^{\frac{1}{\mu}} \rho_{0}^{\frac{2}{\mu}}$. In fact, we consider this inequality only on the support of $\zeta$ contained in the strip $0<t<2^{\frac{1}{k}} \rho^{\frac{2}{k}}$. Therefore the above construction gives an estimate for the $t$-interval of existence of a local solution in terms of the growth of the initial data $u_{1}$.

Theorem 3 If the initial data $u_{1}$ are compactly supported and if

(i) either $N \leq 2$ or

(ii) $N>2, N<\frac{2 r(q+s)+m(r \alpha-s \sigma)}{r(q+s)-m(r+s)}$,

then any nonnegative weak solution to nonhomogeneous problem (1.2), (1.3) has no nontrivial global solution.

\section{Proof of main results}

Proof of Theorem 1 Let $u(x, t)$ be a nontrivial solution of homogeneous problem (1.1), (1.3), and let $\zeta$ be a nonnegative smooth test function. Then

$$
\begin{aligned}
& \int_{0}^{\infty} \int_{R^{N}}|x|^{\alpha}|u|^{q} \zeta\left\|\beta^{\frac{1}{r}}(x)|u|\right\|_{r}^{s} d x d t+\int_{R^{N}} u_{1} \zeta(x, 0) d x \\
& \quad \leq \int_{0}^{\infty} \int_{R^{N}}\left|u \zeta_{t t}\right| d x d t+\int_{0}^{\infty} \int_{R^{N}}\left|u^{m} \Delta \zeta\right| d x d t+\int_{R^{N}} u_{0} \zeta_{t}(x, 0) d x .
\end{aligned}
$$

Now we estimate the right-hand side of inequality (3.1). First, applying the Hölder inequality to the first term in the right-hand side of (3.1), we get

$$
\begin{aligned}
\int_{R^{N}}\left|u \zeta_{t t}\right| d x \leq & \left(\int_{R^{N}}|x|^{\alpha}|u|^{q} \zeta d x\right)^{\frac{1}{\sigma_{1}}}\left(\int_{R^{N}} \beta(x)|u|^{r} d x\right)^{\frac{s}{r \sigma_{1}}} \\
& \times\left(\int_{R^{N}} \beta^{-\frac{s \sigma_{2}}{r \sigma_{1}}}(x)|x|^{-\frac{\alpha \sigma_{2}}{\sigma_{1}}}\left|\zeta_{t t}\right|^{\sigma_{2}} \zeta^{-\frac{\sigma_{2}}{\sigma_{1}}} d x\right)^{\frac{1}{\sigma_{2}}}
\end{aligned}
$$


By the Hölder and Young inequalities again, we obtain

$$
\begin{aligned}
\int_{0}^{\infty} & \int_{R^{N}}\left|u \zeta_{t t}\right| d x d t \\
\leq & \int_{0}^{\infty}\left(\int_{R^{N}}|x|^{\alpha}|u|^{q} \zeta d x\right)^{\frac{1}{\sigma_{1}}}\left(\int_{R^{N}} \beta(x)|u|^{r} d x\right)^{\frac{s}{r \sigma_{1}}} \\
& \times\left(\int_{R^{N}} \beta^{-\frac{s \sigma_{2}}{r \sigma_{1}}}(x)|x|^{-\frac{\alpha \sigma_{2}}{\sigma_{1}}}\left|\zeta_{t t}\right|^{\sigma_{2}} \zeta^{-\frac{\sigma_{2}}{\sigma_{1}}} d x\right)^{\frac{1}{\sigma_{2}}} d t \\
\leq & \left(\int_{0}^{\infty} \int_{R^{N}}|x|^{\alpha}|u|^{q} \zeta \|\left.\beta^{\frac{1}{r}}(x)|u|\right|_{r} ^{s} d x d t\right)^{\frac{1}{\sigma_{1}}} \\
& \times\left(\int_{0}^{\infty}\left(\int_{R^{N}} \beta^{-\frac{s \sigma_{2}}{r \sigma_{1}}}|x|^{-\frac{\alpha \sigma_{2}}{\sigma_{1}}}\left|\zeta_{t t}\right|^{\sigma_{2}} \zeta^{-\frac{\sigma_{2}}{\sigma_{1}}} d x\right)^{\frac{\sigma_{1}^{\prime}}{\sigma_{2}}} d t\right)^{\frac{1}{\sigma_{1}^{\prime}}} \\
\leq & \frac{1}{4} \int_{0}^{\infty} \int_{R^{N}}|x|^{\alpha}|u|^{q} \zeta\left\|\beta^{\frac{1}{r}}(x)|u|\right\|_{r}^{s} d x d t \\
& +C \int_{0}^{\infty}\left(\int_{R^{N}} \beta^{-\frac{s \sigma_{2}}{r \sigma_{1}}}(x)|x|^{-\frac{\alpha \sigma_{2}}{\sigma_{1}}}\left|\zeta_{t t}\right|^{\sigma_{2}} \zeta^{-\frac{\sigma_{2}}{\sigma_{1}}} d x\right)^{\frac{\sigma_{1}^{\prime}}{\sigma_{2}}} d t
\end{aligned}
$$

where

$$
\sigma_{1}=q+s>1, \quad \sigma_{2}=\frac{r(q+s)}{r(q+s)-(r+s)}>1, \quad \frac{1}{\sigma_{1}^{\prime}}=1-\frac{1}{\sigma_{1}} .
$$

We now focus on the second term on the right-hand side of (3.1). Applying similar arguments like for the first term of (3.1), we obtain

$$
\begin{aligned}
\int_{0}^{\infty} \int_{R^{N}}\left|u^{m} \Delta \zeta\right| d x d t \leq & \frac{1}{4} \int_{0}^{\infty} \int_{R^{N}}|x|^{\alpha}|u|^{q} \zeta\left\|\beta^{\frac{1}{r}}(x)|u|\right\|_{r}^{s} d x d t \\
& +C \int_{0}^{\infty}\left(\int_{R^{N}} \beta^{-\frac{s \sigma_{4}}{r \sigma_{3}}}(x)|x|^{-\frac{\alpha \sigma_{4}}{\sigma_{3}}}|\Delta \zeta|^{\sigma_{4}} \zeta^{-\frac{\sigma_{4}}{\sigma_{3}}} d x\right)^{\frac{\sigma_{3}^{\prime}}{\sigma_{4}}} d t
\end{aligned}
$$

where

$$
\sigma_{3}=\frac{q+s}{m}>1, \quad \sigma_{4}=\frac{r(q+s)}{r(q+s)-m(s+r)}>1, \quad \frac{1}{\sigma_{3}^{\prime}}=1-\frac{1}{\sigma_{3}} .
$$

We assume that $\zeta$ is also chosen such that

$$
\int_{R^{N}} u_{0} \zeta_{t}(x, 0) d x=0
$$


By (3.1)-(3.4) we get

$$
\begin{aligned}
& \frac{1}{2} \int_{0}^{\infty} \int_{R^{N}}|x|^{\alpha}|u|^{q} \zeta\left\|\beta^{\frac{1}{r}}(x)|u|\right\|_{r}^{s} d x d t+\int_{R^{N}} u_{1} \zeta(x, 0) d x \\
& \leq C \int_{0}^{\infty}\left(\int_{R^{N}} \beta^{-\frac{s \sigma_{2}}{r \sigma_{1}}}(x)|x|^{-\frac{\alpha \sigma_{2}}{\sigma_{1}}}\left|\zeta_{t t}\right|^{\sigma_{2}} \zeta^{-\frac{\sigma_{2}}{\sigma_{1}}} d x\right)^{\frac{\sigma_{1}^{\prime}}{\sigma_{2}}} d t \\
& \quad+C \int_{0}^{\infty}\left(\int_{R^{N}} \beta^{-\frac{s \sigma_{4}}{r \sigma_{3}}}(x)|x|^{-\frac{\alpha \sigma_{4}}{\sigma_{3}}}|\Delta \zeta|^{\sigma_{4}} \zeta^{-\frac{\sigma_{4}}{\sigma_{3}}} d x\right)^{\frac{\sigma_{3}^{\prime}}{\sigma_{4}}} d t .
\end{aligned}
$$

Now we take $\zeta(x, t)=\varphi\left(\rho^{-2} t^{k}\right) \varphi\left(\rho^{-2}|x|^{\mu}\right)$, where $\varphi \in C_{0}^{2}\left(R_{+}\right)$satisfies $0 \leq \varphi \leq 1$ and

$$
\varphi(x)= \begin{cases}0, & |x| \geq 2 \\ 1, & 0 \leq|x| \leq 1\end{cases}
$$

$\rho$ is a positive parameter, whereas $k>1$ and $\mu>0$ will be determined later on.

Since $\zeta_{t}=k t^{k-1} \rho^{-2} \varphi^{\prime}\left(\rho^{-2} t^{k}\right) \varphi\left(\rho^{-2}|x|^{\mu}\right)$, estimate (3.4) holds. To estimate the right-hand side of (3.5), we consider the change of variables $\rho^{-2} t^{k}=\tau^{k}$ and $\rho^{-2}|x|^{\mu}=|y|^{\mu}$. Then

$$
\begin{aligned}
& \int_{0}^{\infty}\left(\int_{R^{N}} \beta^{-\frac{s \sigma_{2}}{r \sigma_{1}}}(x)|x|^{-\frac{\alpha \sigma_{2}}{\sigma_{1}}}\left|\zeta_{t t}\right|^{\sigma_{2}} \zeta^{-\frac{\sigma_{2}}{\sigma_{1}}} d x\right)^{\frac{\sigma_{1}^{\prime}}{\sigma_{2}}} d t \\
& \quad \leq \rho^{\frac{2}{k}+\left(-\frac{4}{k} \sigma_{2}+\frac{2}{\mu}\left(\frac{s \sigma \sigma_{2}}{r \sigma_{1}}-\frac{\alpha \sigma_{2}}{\sigma_{1}}\right)+\frac{2 N}{\mu}\right) \frac{\sigma_{1}^{\prime}}{\sigma_{2}}} \int_{1}^{2^{\frac{1}{k}}}\left(\int_{|y| \leq 2^{\frac{1}{\mu}}}|y|^{\frac{s \sigma \sigma_{2}}{r \sigma_{1}}-\frac{\alpha \sigma_{2}}{\sigma_{1}}}\left|\zeta_{\tau \tau}\right|^{\sigma_{2}} \zeta^{-\frac{\sigma_{2}}{\sigma_{1}}} d y\right)^{\frac{\sigma_{1}^{\prime}}{\sigma_{2}}} d \tau \\
& \leq C_{1} \rho^{\frac{2}{k}+\left(-\frac{4}{k} \sigma_{2}+\frac{2}{\mu}\left(\frac{s \sigma \sigma_{2}}{r \sigma_{1}}-\frac{\alpha \sigma_{2}}{\sigma_{1}}\right)+\frac{2 N}{\mu}\right) \frac{\sigma_{1}^{\prime}}{\sigma_{2}}}
\end{aligned}
$$

and

$$
\begin{aligned}
& \int_{0}^{\infty}\left(\int_{R^{N}} \beta^{-\frac{s \sigma_{4}}{r \sigma_{3}}}(x)|x|^{-\frac{\alpha \sigma_{4}}{\sigma_{3}}}|\Delta \zeta|^{\sigma_{4}} \zeta^{-\frac{\sigma_{4}}{\sigma_{3}}} d x\right)^{\frac{\sigma_{3}^{\prime}}{\sigma_{4}}} d t \\
& \quad \leq C_{2} \rho^{\frac{2}{k}+\left(-\frac{4}{\mu} \sigma_{4}+\frac{2}{\mu}\left(\frac{s \sigma \sigma_{4}}{r \sigma_{3}}-\frac{\alpha \sigma_{4}}{\sigma_{3}}\right)+\frac{2 N}{\mu}\right) \frac{\sigma_{3}^{\prime}}{\sigma_{4}}},
\end{aligned}
$$

where $C_{1}$ and $C_{2}$ are constants that do not depend $\rho$. From (3.5)-(3.7) we have

$$
\begin{aligned}
& \int_{0}^{\infty} \int_{R^{N}}|x|^{\alpha}|u|^{q} \zeta\left\|\beta^{\frac{1}{r}}(x)|u|\right\|_{r}^{s} d x d t+2 \int_{R^{N}} u_{1} \zeta(x, 0) d x
\end{aligned}
$$

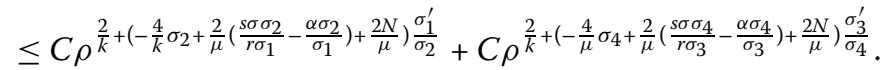

We choose $k$ such that

$$
\begin{aligned}
\frac{2}{k} & +\left(-\frac{4}{k} \sigma_{2}+\frac{2}{\mu}\left(\frac{s \sigma \sigma_{2}}{r \sigma_{1}}-\frac{\alpha \sigma_{2}}{\sigma_{1}}\right)+\frac{2 N}{\mu}\right) \frac{\sigma_{1}^{\prime}}{\sigma_{2}} \\
& =\frac{2}{k}+\left(-\frac{4}{\mu} \sigma_{4}+\frac{2}{\mu}\left(\frac{s \sigma \sigma_{4}}{r \sigma_{3}}-\frac{\alpha \sigma_{4}}{\sigma_{3}}\right)+\frac{2 N}{\mu}\right) \frac{\sigma_{3}^{\prime}}{\sigma_{4}},
\end{aligned}
$$


that is,

$$
\frac{2}{k}=\frac{1}{\mu(q+s-m)}\left((1-m)\left(\frac{s \sigma}{r}-\alpha-\frac{s N}{r}\right)+2(q+s-1)\right) .
$$

Such a choice gives a common value $\gamma$ of the exponents of $\rho$ in (3.8), namely,

$$
\gamma=\frac{1}{\mu(q+s-m)}\left((1+m)\left(\frac{s \sigma}{r}-\alpha-\frac{s N}{r}\right)+2 N(q+s-m)-2(q+s+1)\right) .
$$

The sign of (3.9) does not depend on $\mu>0$ and $k>1$, and

$$
(1-m)\left(\frac{s \sigma}{r}-\alpha-\frac{s N}{r}\right)+2(q+s-1)<2 \mu(q+s-m)
$$

This is ensured by taking $\mu$ large enough.

If $\gamma<0$, then the right-hand side of (3.8) goes to 0 as $\rho$ goes to infinity, which clearly implies that $u$ cannot exist.

If $\gamma=0$, then $\int_{0}^{\infty} \int_{R^{N}}|x|^{\alpha}|u|^{q} \zeta\left\|\beta^{\frac{1}{r}}(x)|u|\right\|_{r}^{s} d x d t<\infty$. We return to inequality (3.1), which in fact reads

$$
\begin{aligned}
& \int_{0}^{\infty} \int_{R^{N}}|x|^{\alpha}|u|^{q} \zeta\left\|\beta^{\frac{1}{r}}(x)|u|\right\|_{r}^{s} d x d t+\int_{R^{N}} u_{1} \zeta(x, 0) d x \\
& \leq C \int_{0}^{\infty} \int_{R^{N}}\left|u \zeta_{t t}\right| d x d t+C \int_{0}^{\infty} \int_{R^{N}}\left|u^{m} \Delta \zeta\right| d x d t \\
& \leq C\left(\int_{\rho^{2} \leq t^{k} \leq 2 \rho^{2}} \int_{\rho^{2} \leq|x|^{\mu} \leq 2 \rho^{2}}|x|^{\alpha}|u|^{q} \zeta\left\|\beta^{\frac{1}{r}}(x)|u|\right\|_{r}^{s} d x d t\right)^{\frac{1}{\sigma_{1}}} \\
& \times\left(\int_{\rho^{2} \leq t^{k} \leq 2 \rho^{2}}\left(\int_{\rho^{2} \leq|x|^{\mu} \leq 2 \rho^{2}} \beta^{-\frac{s \sigma_{2}}{\sigma \sigma_{1}}}|x|^{-\frac{\alpha \sigma_{2}}{\sigma_{1}}}|\zeta t|^{\sigma_{2}} \zeta^{-\frac{\sigma_{2}}{\sigma_{1}}} d x\right)^{\frac{\sigma_{1}^{\prime}}{\sigma_{2}}} d t\right)^{\frac{1}{\sigma_{1}^{\prime}}} \\
& +C\left(\int_{\rho^{2} \leq t^{k} \leq 2 \rho^{2}} \int_{\rho^{2} \leq|x|^{\mu} \leq 2 \rho^{2}}|x|^{\alpha}|u|^{q} \zeta\left\|\beta^{\frac{1}{r}}(x) \mid u\right\|_{r}^{s} d x d t\right)^{\frac{1}{\sigma_{3}}} \\
& \times\left(\int_{\rho^{2} \leq t^{k} \leq 2 \rho^{2}}\left(\int_{\rho^{2} \leq|x|^{\mu} \leq 2 \rho^{2}} \beta^{-\frac{s \sigma_{4}}{r \sigma_{3}}}(x)|x|^{-\frac{\alpha \sigma_{4}}{\sigma_{3}}}|\Delta \zeta|^{\sigma_{4}} \zeta^{-\frac{\sigma_{4}}{\sigma_{3}}} d x\right)^{\frac{\sigma_{3}^{\prime}}{\sigma_{4}}} d t\right)^{\frac{1}{\sigma_{3}^{\prime}}} .
\end{aligned}
$$

However, $\int_{0}^{\infty} \int_{R^{N}}|x|^{\alpha}|u|^{q} \zeta\left\|\beta^{\frac{1}{r}}(x)|u|\right\|_{r}^{s} d x d t<\infty$ implies that

$$
\lim _{\rho \rightarrow \infty} \int_{\rho^{2} \leq t^{k} \leq 2 \rho^{2}} \int_{\rho^{2} \leq|x|^{\mu} \leq 2 \rho^{2}}|x|^{\alpha}|u|^{q} \zeta\left\|\beta^{\frac{1}{r}}(x)|u|\right\|_{r}^{s} d x d t=0 .
$$

This infers that

$$
\int_{0}^{\infty} \int_{R^{N}}|x|^{\alpha}|u|^{q} \zeta\left\|\beta^{\frac{1}{r}}(x)|u|\right\|_{r}^{s} d x d t=0
$$


Now the assumption $\gamma \leq 0$ means

$$
\begin{gathered}
2 r(q+s)(N-1) \leq(1+m)(r \alpha-s \sigma+s N)+2 r(1+m N) \\
\quad \Leftrightarrow \quad N \leq \frac{2 r(q+s+1)+(1+m)(r \alpha-s \sigma)}{2 r(q+s-m)-(1+m) s} .
\end{gathered}
$$

Note if $N=1$, then (3.10) is obviously fulfilled.

Remark 2 The integrability assumption on $u_{1}$ can be relaxed by replacing the sign condition by the following weaker one:

$$
\liminf _{\rho \rightarrow \infty} \int_{R^{N} \cap\left\{|x| \leq 2^{\frac{1}{\mu}} \rho^{\frac{2}{\mu}}\right\}} u_{1} \zeta(x, 0) d x \geq 0
$$

Proof of Theorem 3 Following the proof of Theorem 1, we obtain the following estimate analogous to the estimate in Theorem 2:

$$
\begin{aligned}
& \int_{0}^{2^{\frac{1}{k}} \rho^{\frac{2}{k}}} \int_{R^{N} \cap\left\{|x| \leq 2^{\frac{1}{\mu}} \rho^{\frac{2}{\mu}}\right\}}|x|^{\alpha}|u|^{q} \zeta\left\|\beta^{\frac{1}{r}}(x)|u|\right\|_{r}^{s} d x d t+\int_{R^{N} \cap\left\{|x| \leq 2^{\frac{1}{\mu}} \rho^{\frac{2}{\mu}}\right\}} u_{1} \zeta(x, 0) d x \\
& \quad+\int_{R^{N} \cap\left\{|x| \leq 2^{\frac{1}{\mu}} \rho^{\frac{2}{\mu}}\right\}} \omega(x) \zeta(x, 0) d x \leq C \rho^{\frac{2}{k}+\left(-\frac{4}{\mu} \sigma_{4}+\frac{2}{\mu}\left(\frac{s \sigma \sigma_{4}}{r \sigma_{3}}-\frac{\alpha \sigma_{4}}{\sigma_{3}}\right)+\frac{2 N}{\mu}\right) \frac{\sigma_{3}^{\prime}}{\sigma_{4}}}
\end{aligned}
$$

whence

$$
c_{\omega} \rho^{\frac{2}{k}} \leq \int_{0}^{2^{\frac{1}{k}} \rho^{\frac{2}{k}}} \int_{R^{N} \cap\left\{|x| \leq 2^{\frac{1}{\mu}} \rho^{\frac{2}{\mu}}\right\}} \omega(x) \zeta d x d t \leq C \rho^{\frac{2}{k}+\left(-\frac{4}{\mu} \sigma_{4}+\frac{2}{\mu}\left(\frac{s \sigma \sigma_{4}}{r \sigma_{3}}-\frac{\alpha \sigma_{4}}{\sigma_{3}}\right)+\frac{2 N}{\mu}\right) \frac{\sigma_{3}^{\prime}}{\sigma_{4}}}
$$

if $\rho$ is such that

$$
\int_{R^{N} \cap\left\{|x| \leq 2^{\frac{1}{\mu}} \rho^{\frac{2}{\mu}}\right\}} \omega(x) \zeta(x, 0) d x \geq c_{\omega} \equiv \text { const }>0 .
$$

Assuming that

$$
\gamma_{1}=\left(-\frac{4}{\mu} \sigma_{4}+\frac{2}{\mu}\left(\frac{s \sigma \sigma_{4}}{r \sigma_{3}}-\frac{\alpha \sigma_{4}}{\sigma_{3}}\right)+\frac{2 N}{\mu}\right) \frac{\sigma_{3}^{\prime}}{\sigma_{4}}<0,
$$

we obtain a contradiction by letting $\rho \rightarrow \infty$. Next, from (3.13) we have

$$
\begin{gathered}
r(q+s)(N-2)<m(r \alpha-s \sigma)+m N(r+s) \\
\Leftrightarrow \quad N<\frac{2 r(q+s)+m(r \alpha-s \sigma)}{r(q+s)-m(r+s)} .
\end{gathered}
$$

Notice that if $N \leq 2$, then (3.14) is obviously fulfilled. The proof of Theorem 3 is completed. 
Funding

The work of the second author (Fang) was supported by the Natural Science Foundation of Shandong Province of China (No. ZR2019MA072) and the Fundamental Research Funds for the Central Universities (No. 201964008).

\section{Abbreviations}

Not applicable.

Availability of data and materials

Data sharing is not applicable to this paper as no data sets were generated or analyzed during the current study.

\section{Competing interests}

The authors declare that they have no competing interests.

\section{Authors' contributions}

Both authors contributed equally and significantly in writing this paper. Both authors read and approved the final manuscript.

\section{Author details}

${ }^{1}$ School of Mathematical and Computer Science, Shanxi Normal University, Linfen, Shanxi, 041004, P.R. China. ${ }^{2}$ School of Mathematical Sciences, Ocean University of China, Qingdao 266100, P.R. China.

\section{Publisher's Note}

Springer Nature remains neutral with regard to jurisdictional claims in published maps and institutional affiliations.

Received: 30 June 2020 Accepted: 20 August 2021 Published online: 03 September 2021

\section{References}

1. Galaktionov, V.A., Vazquez, J.L.: Continuation of blowup solutions of nonlinear heat equations in several space dimensions. Commun. Pure Appl. Math. 50, 1-67 (1997)

2. John, F: Blow-up of solutions of nonlinear wave equations in three space dimensions. Proc. Natl. Acad. Sci. USA 76(4), 1559-1560 (1979)

3. Glassey, R.T.: Finite-time blow-up for solutions of nonlinear wave equations. Math. Z. 177(3), 323-340 (1981)

4. Strauss, W.A., Tsutaya, K.: Existence and blow up of small amplitude nonlinear waves with a negative potential. Discrete Contin. Dyn. Syst., Ser. B 3(2), 175-188 (1997)

5. Tsutaya, K.: Global existence and the life span of solutions of semilinear wave equations with data of non compact support in three space dimensions. Funkc. Ekvacioj 37(1), 1-18 (1994)

6. Pohozaev, S., Veron, L.: Blow-up results for nonlinear hyperbolic inequalities. Ann. Sc. Norm. Super. Pisa, Cl. Sci. 29(2), 393-420 (2000)

7. Guedda, M.: Local and global nonexistence of solutions to nonlinear hyperbolic inequalities. Appl. Math. Lett. 16(4), 493-499 (2003)

8. Laptev, G.G.: Absence of solutions of differential inequalities and systems of hyperbolic type in conic domains. Izv. Math. 66(6), 1147-1170 (2002)

9. Mitidieri, E., Pokhozhaev, S.I.: The absence of global positive solutions to quasilinear elliptic inequalities. Dokl. Math 57(2), 250-253 (1998)

\section{Submit your manuscript to a SpringerOpen ${ }^{\circ}$ journal and benefit from:}

- Convenient online submission

- Rigorous peer review

- Open access: articles freely available online

- High visibility within the field

- Retaining the copyright to your article 